\title{
The age at onset in Multiple Sclerosis is associated with patient's prognosis
}

\author{
Cierny $\mathrm{D}^{1}$, Lehotsky $\mathrm{J}^{2}$, Hanysova $\mathrm{S}^{3}$, Michalik $\mathrm{J}^{4}$, Kantorova $\mathrm{E}^{4}$, Sivak $\mathrm{S}^{4}$, Kurca $\mathrm{E}^{4}$, \\ Dobrota $\mathrm{D}^{1,3}$, Jesenska $\mathrm{L}^{1}$
}

Department of Clinical Biochemistry, Jessenius Faculty of Medicine, Comenius University in Bratislava and University Hospital Martin, Martin, Slovakia. lujesenska@unm.sk

\begin{abstract}
BACKGROUND: The causes of the individual differences in the rate of disability progression in multiple sclerosis (MS) are still not completely clear. According to the long-term prognosis of MS patients, the search for new valuable prognostic markers of "benign" or "malign" MS is necessary.

OBJECTIVES: Our aim was to assess the possible association of MS onset age with the disease disability progression rate in Slovak patients with MS.

METHODS: By the unique pattern of evaluation of disability progression rate using Multiple Sclerosis Severity Score (MSSS), each of 270 MS patients was defined as slow-progressing, mid-rate progressing or rapidly progressing.

RESULTS: We found a significant differences in the age at onset between MS patients with different rate of disability progression ${ }_{(p(K-W)<0,00005)}$. The faster was a disability progression assessed by MSSS score, the higher was the MS onset age.

CONCLUSION: We showed for the first time in Central European Slovak population that MS onset age is an early marker that is in the positive correlation with disease disability progression rate, evaluated by MSSS score. We conclude that relapsing-remitting MS patients older at clinical onset have a higher risk of unfavorable prognosis (Tab. 2, Fig. 1, Ref. 21). Text in PDF www.elis.sk.

KEY WORDS: multiple sclerosis, prognosis, disability progression, MSSS, age at onset.
\end{abstract}

\section{Introduction}

Sclerosis multiplex (multiple sclerosis, MS) is a chronic autoimmune demyelinating disease of the central nervous system (CNS), affecting predominantly young adults. The main etiopathogenetic trait of the disease is the formation and activation of autoreactive clones of T-lymphocytes. After penetration to the CNS, these T-cells trigger a cascade of processes leading to demyelination and axonal injury, and subsequent neurological damage (1). The disease is most commonly present in its relapsing-remitting form with clinical attacks of neurological dysfunction (relapses),

${ }^{1}$ Department of Clinical Biochemistry, Jessenius Faculty of Medicine, Comenius University in Bratislava and University Hospital Martin, Martin, Slovakia, ${ }^{2}$ Department of Medical Biochemistry and BioMed, Jessenius Faculty of Medicine in Martin, Comenius University in Bratislava, Martin, Slovakia, ${ }^{3}$ Department of Medical Biochemistry, Jessenius Faculty of Medicine in Martin, Comenius University in Bratislava, Martin, Slovakia, and ${ }^{4}$ Department of Neurology, Jessenius Faculty of Medicine, Comenius University in Bratislava and University Hospital Martin, Martin, Slovakia

Address for correspondence: L. Jesenska, Mgr, Department of Clinical Biochemistry, Jessenius Faculty of Medicine in Martin, Comenius University in Bratislava and University hospital Martin, Kollarova 2, SK-036 59 Martin, Slovakia.

Phone: +421.907309565

Acknowledgement: This study was supported by the project „Centre for Translational Medicine“, ITMS code: 26220220021. characterized by an increased local and general inflammatory activity (2). Recent data indicated that the process of neurodegeneration runs independently from neuroinflammation even in the early stages of the disease and to a large degree it correlates with a progression of disability (3). Although multiple sclerosis is still an un-curable disease, immunomodulatory Disease Modifying Drugs (DMD) administered to the affected individuals can successfully reduce the number of relapses, ameliorate the disease course and improve the quality of life.

Interestingly, according to the clinical course of MS, we can distinguish „benign MS“ characterized by slow disability progression and on the other hand, the „,malign MS“ with the rapid disability progression, possibly resulting in inability to walk (4). The causes of these differences in disease progression are still not clear and it is necessary to search for new prognostic markers, that could help to identify the patients predisposed to an increased risk of rapid disability progression. In these patients, early and appropriate therapeutic intervention is important to slow down neurological damage. Good examples of these eligible markers with promising practical application, significantly correlating with the disease characteristics, are gene polymorphisms, together with other epigenetic, biochemical and immunological markers (5-8).

Apart from these markers, the onset age of MS, that is directly available at the day of MS diagnosis has been recently discussed to be one of the possible factors associated with MS prognosis. 
Several studies in different populations suggested for the association of higher MS onset age with time to reach certain EDSS score milestones, but not with consistent results (9-16). In our study in Slovak MS patients, we tried to identify whether MS onset age is associated with the disability progression rate, evaluated by the unique pattern of MS patients stratification, based on Multiple Sclerosis Severity Score (MSSS), that has not been previously used in the study of this type.

\section{Group of participants}

Our study included 270 patients with clinically diagnosed MS (204 women, 66 men). The female to male ratio was $3: 1$, as in natural MS population. The mean age of MS patients was 41.3 \pm 10.8 years. The study was approved by the ethical committee of the Comenius University in Bratislava, Jessenius Faculty of Medicine in Martin. All individuals participating in our study gave an informed consent. Clinically definitive MS diagnosis was established according to the McDonald's criteria in The Centre for Demyelinating Diseases at the Clinic of Neurology, Jessenius Faculty of Medicine, Comenius University in Bratislava and University Hospital Martin, Slovakia. All individuals involved in the study were Slovak people living in the north-central part of Slovakia.

The rate of disability progression was evaluated by the MSSS score, that was obtained from the Global MSSS table, using each patient's EDSS score and disease duration data (17). For the stratification of MS patients, we used a unique pattern, that was designed by ourselves according to the experience from clinical practice. We classified the MS patients with MSSS $<3$ as slow progressing (MS-1, $\mathrm{n}=82$ ), with MSSS $=3-6$ as mid-rate progressing (MS$2, \mathrm{n}=130$ ) and with MSSS $>6$ as rapidly progressing (MS-3, $\mathrm{n}=$ 58). Clinical characteristics of the whole MS patients group and subgroups of patients with different rates of disability progression are shown in Table 1.

\section{Statistical analysis}

Statistical analysis was performed by SVS 7 software (SNP \& Variation Suite v7.6.11, Golden Helix, Bozeman, Montana, USA). To estimate the significance of differences between the subgroups of categorical variables with more than 2 categories, we used the
Kruskal-Wallis test. Statistical significance level was considered to be two-tailed $\mathrm{p} \leq 0.05$ in all tests performed.

\section{Results}

In our study, we found that the onset age of MS significantly correlated with the disability progression rate evaluated by MSSS score $_{(\mathrm{p}(\mathrm{K}-\mathrm{W})<0.00005)}$. The data are shown in the Table 2 and Figure 1. Interestingly, in the subgroup of MS patients with a slow disability progression, the mean onset age was $25.4 \pm 8.3$ years, in the mid-rate progressing MS patients, it was $29.7 \pm 9.7$ years, while in the rapidly progressing MS patients, it was $34.6 \pm 10.5$ years.

\section{Discussion}

MS is a complex disease that develops usually in young adults before the age of 30 , but there are also individuals with late onset. MS is characterized by large interindividual differences in the disease course and progression, which could be the result of interaction of many genetic, environmental or other factors. Nowadays, there is still no relevant panel of prognostic markers that could help to identify the individuals predisposed to a rapid disability progression.

The results of our study showed that the higher was the MS onset age, the faster was a disease disability progression evaluated by MSSS score ${ }_{(\mathrm{p}(\mathrm{K}-\mathrm{W})<0.00005)}$. It is necessary to point out, that in our study, we used the unique pattern of MS patients stratification, that was based on MSSS score, considering individually the patient's disability and disease duration in any stage of the disease and thus defining slow-progressing, mid-rate progressing and rapidly progressing MS. Until now, no such study had been yet made in Central European Slovak population. Only several studies that suggested an association of MS onset age with disability progression have been performed in different populations. However, to measure the disability progression, the majority of authors used the time to reach certain EDSS scores or they related prognostic markers to the form of the disease.

Tremlett et al (9) evaluated MS disability progression by the time it took to reach EDSS score of 6.0 in a group of $2837 \mathrm{MS}$ patients from Canada. Similarly, to our results, they found that the younger onset age predicted a slower progression. Interestingly,

Tab. 1. Clinical characteristics of the MS patients.

\begin{tabular}{|c|c|c|c|c|}
\hline & \multirow{2}{*}{$\begin{array}{l}\text { MS patients } \\
\quad(\mathrm{n}=270)\end{array}$} & \multicolumn{3}{|c|}{ Progression of disability - MS subgroups } \\
\hline & & $\begin{array}{c}\text { Slow - MS-1 } \\
(\mathrm{n}=82,30.4 \%)\end{array}$ & $\begin{array}{l}\text { Mid-rate }- \text { MS-2 } \\
(\mathrm{n}=130,48.1 \%)\end{array}$ & $\begin{array}{l}\text { Rapid - MS-3 } \\
(\mathrm{n}=58,21.5 \%)\end{array}$ \\
\hline Age of the first symptoms (years) & $29.2 \pm 10.0$ & $25.4 \pm 8.3$ & $29.7 \pm 9.7$ & $34.6 \pm 10.5$ \\
\hline Disease course (years) & $12.1 \pm 7.1$ & $14.6 \pm 7.0$ & $11.6 \pm 7.3$ & $10.2 \pm 6.5$ \\
\hline EDSS score (points) & $3.5 \pm 1.6$ & $2.2 \pm 0.9$ & $3.7 \pm 1.2$ & $5.3 \pm 1.6$ \\
\hline MSSS (points) & $4.25 \pm 2.08$ & $1.92 \pm 0.74$ & $4.36 \pm 0.77$ & $7.30 \pm 1.00$ \\
\hline \multicolumn{5}{|l|}{ MS phenotype } \\
\hline Relapsing-remitting & $\mathrm{n}=230(85.2 \%)$ & $\mathrm{n}=81(98.8 \%)$ & $\mathrm{n}=114(87.7 \%)$ & $\mathrm{n}=35(60.3 \%)$ \\
\hline Secondary progressive & $\mathrm{n}=40(14.8 \%)$ & $\mathrm{n}=1(1.2 \%)$ & $\mathrm{n}=16(12.3 \%)$ & $\mathrm{n}=23(39.7 \%)$ \\
\hline
\end{tabular}

MS - multiple sclerosis, EDSS - expanded disability status scale, MSSS - multiple sclerosis severity score 
Tab. 2. Age at onset in MS and the rate of disease disability progression evaluated by MSSS score.

\begin{tabular}{lcccc}
\hline & $\begin{array}{c}\text { MS-1 } \\
(\mathrm{n}=82)\end{array}$ & $\begin{array}{c}\text { MS-2 } \\
(\mathrm{n}=130)\end{array}$ & $\begin{array}{c}\text { MS-3 } \\
(\mathrm{n}=58)\end{array}$ & $\begin{array}{c}\text { MS-1 vs MS-2 vs. MS-3 } \\
\mathrm{p}_{(\mathrm{K}-\mathrm{W})}\end{array}$ \\
\cline { 1 - 4 } $\begin{array}{l}\text { Age at onset } \\
\text { (years) }\end{array}$ & $25.4 \pm 8.3$ & $29.7 \pm 9.7$ & $34.6 \pm 10.5$ & $<0.00005$ \\
\cline { 1 - 2 } $\begin{array}{l}\text { MSSS } \\
\text { (points) }\end{array}$ & $1.92 \pm 0.74$ & $4.36 \pm 0.77$ & $7.30 \pm 1.00$ & \\
\hline
\end{tabular}

MS-1 - slow progressing multiple sclerosis, MS-2 - mid-rate progressing multiple sclerosis, MS-3 - rapidly progressing multiple sclerosis, MSSS - multiple sclerosis severity score

they demonstrated that worse disease outcome was associated neither with older onset age nor with male sex. Trojano et al (10) in the group of 1463 MS patients from Apulian $(n=1039)$ and Sardinian $(n=424)$ regions of Italy observed faster rates of disease progression in older patients, interestingly with a greater effect of the current age than the age at disease onset. They found the time necessary to reach EDSS 4.0 or EDSS 6.0 to be significantly longer in MS patients that were 20-35 years old than in patients aged 36-50 and 51-65. Controversially, the results of their study suggested that MS progression was independent of age at disease onset and patients with early onset would approach the rate of disability of those with late onset when they reach a similar age.

Myhr et al (11) in the group of 220 MS patients from Western Norway assessed the MS progression by the time elapsing before reaching EDSS $>6.0$. In relapsing-remitting MS, they identified lower age at onset as a clinical positive prognostic marker of the disability progression. Higher onset age was identified to be a clinical negative prognostic marker of MS disability progression in the patients with primary progressive MS.

The study of Alroughani et al (12) was performed among 903 MS patients from Kuwait. They showed that late onset MS pa-

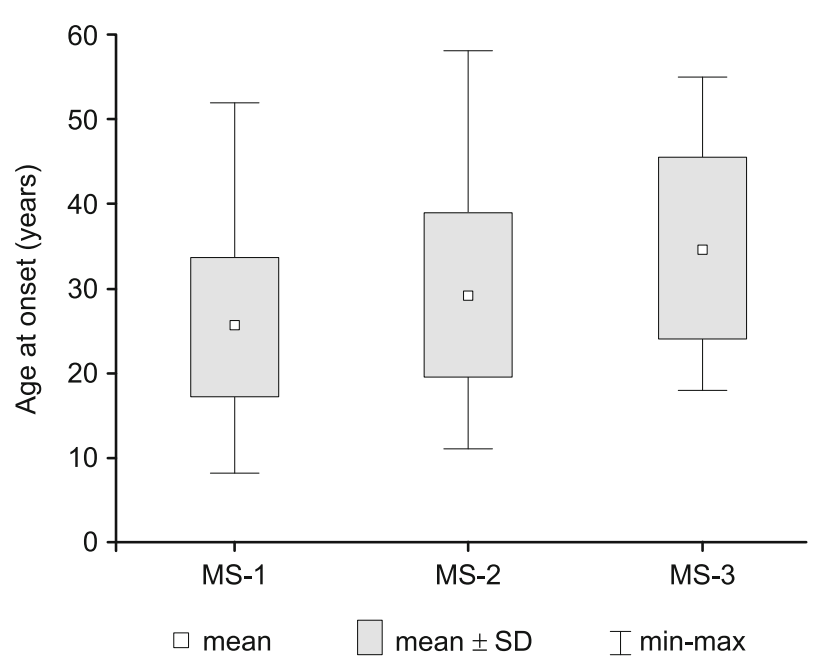

Fig. 1. Age at onset in the subgroups of MS patients with different rate of disability progression. MS-1 - slow progressing multiple sclerosis, MS-2 - mid-rate progressing multiple sclerosis, MS-3 - rapidly progressing multiple sclerosis. tients had significantly shorter time to reach EDSS 6.0 compared to younger MS patients, with higher risk influenced by the male gender and spinal cord presentation at onset. Also Cournu-Rebeix et al (13) in the group of 651 MS patients from France observed that time to reach EDSS score of 6.0 was significantly shorter for patients with age at onset higher than 40 years than for others, as well as in primary progressive patients when compared to secondary progressive and relapsing-remitting patients.

Leray et al (14) correlated age cut-offs with EDSS 3.0 and EDSS 6.0 milestones in 2054 MS patients from West France. In relapsing-remitting MS, they found the younger age at onset to be associated with a slower disability progression before reaching EDSS 3.0. Also, the median times from clinical onset of MS to reach EDSS 6.0 were found to decrease with increasing age at onset. In contrast, in progressive onset multiple sclerosis, age at onset did not influence disability progression.

Scalfari et al (15) in a group of 1023 MS patients from United Kingdom investigated the influence of age at onset in MS on attainment of severe disability levels characterized by EDSS 6.0, EDSS 8.0 and EDSS 10.0. They found, that patients younger at onset of the relapsing-remitting MS had a lower hazard of reaching EDSS levels and of entering the secondary progressive phase. In their study, the patients with onset at age 40 and at age 50 had twice and three times higher risk of converting to secondary progressive MS compared to the onset age of 20. Also, the risk of having a primary progressive disease course was found to be increased with age at onset.

In another study of a group of 1844 MS patients from France, Confavreux et Vukusic (16) investigated possible factors influencing the time to reach the EDSS score of 4.0, 6.0 and 7.0. The cohort consisted of mainly Caucasians with some admixture from the former French colonies in North Africa. They found MS prognosis to be age and sex dependent. The younger age at clinical onset was showed to be associated with a younger age of assignment of disability EDSS milestones and irreversible disability was found to be reached at a younger age in males than in females.

Amato et al (18) were investigating the prognostic markers according to the time to reach the EDSS 4.0 and 6.0 in a group of $224 \mathrm{MS}$ patients from Italy. In their study, the association with age at onset was not found, but they identified many other predictors of poor outcome, mainly the higher EDSS score at the disease onset and a primary progressive course.

Considering our results and the results of the previous studies, it seems that the age at onset of MS have an impact on the disease disability progression rate in Slovaks, but also in the other populations. The association is possibly influenced by many other factors, including current age, sex, disease course at onset etc. We do not exclude the possible impact of the patient's ethnicity or geographical location of residence.

We may only speculate what could be the principal cause of these findings. The neurological disability in MS, that can be to some degree compensated, is a result of several processes including abnormal focal and general inflammatory process related to autoimmune demyelination, axonal injury and neurodegeneration. We suppose that apart from all these processes, inherently present 
in MS, the rapid disability progression found in MS patients with later onset may be the result of the age-related deterioration of physiological functions and compensatory mechanisms. They can be age-related decrease in CNS remyelinating efficiency caused by an insufficient oligodendrocyte progenitor recruitment to the damaged site or their failure to differentiate into remyelinating oligodendrocytes (19); age-related cerebral mitochondrial dysfunction and neuronal apoptosis (20); deterioration of brain energy production and fosfolipid metabolism (21) or other age-related changes in immunological reactivity or in ability to compensate for oxidative stress (16).

According to our knowledge, this is the first study assessing the association between MS onset age and disability progression rate evaluated by MSSS score in Central European Slovak population. The previous studies that were performed in different populations associated the MS onset age with the time to reach EDSS score milestones. In our study, we have showed for the first time that MS onset age was associated with the disability progression rate, evaluated by the unique pattern using Multiple Sclerosis Severity Score, the most precise way how to assess MS disability progression in any stage of the disease. In our study, we identified the higher MS onset age to be a clinical negative prognostic factor that correlated with a probability of more rapid disease disability progression in Slovak MS patients. We would like to emphasize that the age at onset is a very simply measurable factor, which is non-invasive and available directly at the day of diagnosis with no extra costs. Therefore, we propose the onset age to be added to other clinical and paraclinical markers that can be used in individual management of MS patients in the early prediction of disease disability progression rate.

\section{References}

1. Compston A, Coles A. Multiple sclerosis. Lancet 2008; 372 (9648): $1502-1517$.

2. Murta V, Ferrari CC. Influence of Peripheral inflammation on the progression of multiple sclerosis: evidence from the clinic and experimental animal models. Mol Cell Neurosci 2013; 53: 6-13.

3. Levin MC, Douglas JN, Meyers L, Lee S, Shin Y, Gardner LA. Neurodegeneration in multiple sclerosis involves multiple pathogenic mechanisms. Degener Neurol Neuromusc Dis 2014; 2014 (4): 49-63.

4. Jedlicka P. Onemocnění bílé hmoty mozkomíšní. In: Jedlička P, Keller O (Eds). Speciální neurologie. Praha: Galén, 2005: 203-212.

5. Cierny D, Michalik J, Kurca E, Dobrota D, Lehotsky J. FokI vitamin $\mathrm{D}$ receptor gene polymorphism in association with multiple sclerosis risk and disability progression in Slovaks. Neurol Res 2015; 37 (4): 301-308.

6. Cierny D, Hanysová S, Michalik J, Kantorova E, Kurca E, Skerenova M, Lehotsky J. Genetic variants in interleukin 7 receptor $\alpha$ chain (IL-
7Ra) are associated with multiple sclerosis risk and disability progression in Central European Slovak population. J Neuroimmunol 2015; 282: 80-84.

7. Čierny D, Michalik J, Škereňová M et al. ApaI, BsmI and TaqI VDR gene polymorphisms in association with multiple sclerosis in Slovaks. Neurol Res 2016; 38 (8): 678-684.

8. Michalik J, Cierny D, Kantorova $\mathbf{E}$ et al. The association of HLADRB1 and HLA-DQB1 alleles with genetic susceptibility to multiple sclerosis in the Slovak population. Neurol Res 2015; 37 (12): 1060-1067.

9. Tremlett H, Paty D, Devonshire V. Disability progression in multiple sclerosis is slower than previously reported. Neurology 2006; 66 (2): $172-177$.

10. Trojano M, Liguori M, Bosco Zimatore G et al. Age-related disability in multiple sclerosis. Ann Neurol 2002; 51 (4): 475-480.

11. Myhr KM, Riise T, Vedeler C, Nortvedt MW, Grønning R, Midgard R, Nyland HI. Disability and prognosis in multiple sclerosis: demographic and clinical variables important for the ability to walk and awarding of disability pension. Multiple Sclerosis 2001; 7 (1): 59-65.

12. Alroughani R, Akhtar S, Ahmed S, Behbehani R, Al-Hashel J. Is Time to Reach EDSS 6.0 Faster in Patients with Late-Onset versus YoungOnset Multiple Sclerosis? PLoS One 2016; 11 (11): e0165846.

13. Cournu-Rebeix I, Génin E, Leray $\mathbf{E}$ et al. HLA-DRB1*15 allele influences the later course of relapsing remitting multiple sclerosis. Genes Immunity 2008; 9 (6): 570-574.

14. Leray E, Yaouanq J, Le Page E, Coustans M, Laplaud D, Oger J, Edan G. Evidence for a two-stage disability progression in multiple sclerosis. Brain 2010; 133 (7): 1900-1913.

15. Scalfari A, Neuhaus A, Daumer M, Ebers GC, Muraro PA. Age and disability accumulation in multiple sclerosis. Neurology 2011; 77 (13): 1246-1252.

16. Confavreux C, Vukusic S. Age at disability milestones in multiple sclerosis. Brain 2006; 129 (3): 595-605.

17. Roxburgh RH, Seaman SR, Masterman T et al. Multiple sclerosis severity score. Using disability and disease duration to rate disease severity. Neurology 2005; 64 (7): 1144-1151.

18. Amato MP, Ponziani G. A prospective study on the prognosis of multiple sclerosis. Neurological Sciences 2000; 21 (Suppl 2): S831-838.

19. Sim FJ, Zhao C, Penderis J, Franklin RJ. The age-related decrease in CNS remyelination efficiency is attributable to an impairment of both oligodendrocyte progenitor recruitment and differentiation. J Neurosci 2002; 22 (7): 2451-2459.

20. Tatarkova Z, Kovalska M, Timkova V, Racay P, Lehotsky J, Kaplan P. The Effect of Aging on Mitochondrial Complex I and the Extent of Oxidative Stress in the Rat Brain Cortex. Neurochem Res 2016; 41 (8): 2160-2172.

21. Forester BP, Berlow YA, Harper DG et al. Age-related changes in brain energetics and phospholipid metabolism. NMR Biomed 2010; 23 (3): $242-250$. 